\title{
Reduction in total radiation exposure using X-ray image guidance illustrated in a patient receiving pituitary radiotherapy
}

Frederic Brochu ${ }^{1,2}$, Marina Romanchikova ${ }^{3}{ }^{\star}$, Simon J Thomas ${ }^{3}$, Andrew CF Hoole ${ }^{3}$, David $\mathrm{J}_{\text {Noble }}{ }^{2}$, Michael PD Simmons ${ }^{1}$, Mark Gurnell ${ }^{4}$, Michael A Parker ${ }^{1}$, Neil G Burnet ${ }^{2 \pi} \S$

1. University of Cambridge, Department of Physics, Cavendish Laboratory, JJ Thomson Avenue, Cambridge, CB3 OHE, UK;

2. University of Cambridge Department of Oncology, Cambridge Biomedical Campus, Addenbrooke's Hospital, Hills Road, Cambridge, CB2 0QQ, UK;

3. Department of Medical Physics and Clinical Engineering, Addenbrooke's Hospital, Cambridge, CB2 0QQ, UK;

4. Metabolic Research Laboratories, Wellcome Trust-MRC Institute of Metabolic Science, University of Cambridge and National Institute for Health Research, Cambridge Biomedical Research Centre, Addenbrooke's Hospital, Cambridge, UK, CB2 0QQ;

${ }^{\mathbb{}}$ Author for correspondence

*Current address: National Physical Laboratory, Maxwell Centre, JJ Thomson Avenue, Cambridge, CB3 OHE.

$\S$ Address from 1st February 2018: Manchester Cancer Research Centre, The University of Manchester, 555 Wilmslow Road, Manchester, M20 4GJ. 


\section{Dear Editor,}

Choudhury et al [1] discuss the considerable promise of MR-based image guidance, and we strongly support the implementation and evaluation of this exciting technology. However, for the foreseeable future most patients will be treated using X-ray-based image guidance (IGRT). Although the importance of IGRT is well recognised [2], there is evidence that full use of IGRT capability has not yet been achieved [2, 3], partly because of fear of the radiation dose.

IGRT is used primarily to ensure accurate dose delivery to the target. Once data on target positioning data has been obtained, it is sometimes possible to reduce planning target volume (PTV) margins. The figure shows the case of a patient with recurrent non-functioning pituitary adenoma. His original plan was compared to an equivalent plan with reduced PTV margin ( $5 \mathrm{~mm}$, volume $34.6 \mathrm{~cm}^{3}$, to $3 \mathrm{~mm}$, volume $24.7 \mathrm{~cm}^{3}$ ), as would be used now [4]. The dose from daily cone beam CT (CBCT) image guidance scans was added to the $3 \mathrm{~mm}$ plan [5]. The dose difference map was produced by subtraction and clearly shows bands of unnecessary dose.

X-ray IGRT carries a 'cost' of radiation exposure. However, reduction of the PTV margin reduces the overall radiation burden, as eloquently described by van Herk a decade ago [6]. Here, the $5 \mathrm{~mm}$ PTV plan with no IGRT would deliver an estimated absorbed energy of 24.97 J. The use of a $3 \mathrm{~mm}$ margin, combined with CBCT dose, delivers $21.59 \mathrm{~J}$ (difference $3.38 \mathrm{~J}$ or $13.5 \%)$. The additional energy from CBCT was estimated at $0.42 \mathrm{~J}$ ( $1.9 \%$ of the total). Use of IGRT with a lower frequency, permitting some PTV margin reduction, still leads to reduced total patient dose.

Thus, the use of X-ray IGRT can lead to a reduction in total radiation burden, which should actively encourage its use.

\section{Acknowledgement:}

We kindly thank Dr Gustav Meedt from Elekta for helpful discussions. FMB was funded by a STFC Global Challenge Concept grant. MG and NGB are supported by the NIHR Cambridge Biomedical Research Centre. DJN is supported by Addenbrooke's Charitable Trust and Cancer Research UK. We are grateful to Jane Sales \& Mala Jayasundera for help in preparing the manuscript. 


\section{References}

1. Choudhury A, Budgell G, MacKay R, Falk S, Faivre-Finn C, Dubec M, van Herk M, McWilliam A. The Future of Image-guided Radiotherapy. Clin Oncol (R Coll Radiol). 2017; 29(10): 662-666.

2. National Radiotherapy Implementation Group Report. Image guided radiotherapy (IGRT): Guidance for implementation and use. Department of Health, 2012. https://www.sor.org/sites/default/files/documentversions/National\%20Radiotherapy\%20Implementation\%20Group\%20Report\%20IGRT\% 20Final.pdf (accessed 18.5.17)

3. Nabavizadeh N, Elliott DA, Chen Y, Kusano AS, Mitin T, Thomas CR Jr, Holland JM. Image Guided Radiation Therapy (IGRT) Practice Patterns and IGRT's Impact on Workflow and Treatment Planning: Results From a National Survey of American Society for Radiation Oncology Members. Int J Radiat Oncol Biol Phys. 2016 Mar 15; 94(4): 850-7.

4. Burnet NG, Adams EJ, Fairfoul J, Tudor GSJ, Hoole ACF, Routsis, DS Dean JC, Kirby RD, Cowen M, Russell SG, Rimmer YL, Thomas SJ. Practical aspects of implementation of helical tomotherapy for intensity-modulated and image-guided radiotherapy. Clin Oncol. 2010; 22(4): 294-312.

5. Brochu FM1, Burnet NG, Jena R, Plaistow R, Parker MA, Thomas SJ. Geant4 simulation of the Elekta XVI kV CBCT unit for accurate description of potential late toxicity effects of image-guided radiotherapy. Phys Med Biol. 2014 Dec 21;59(24):7601-8.

6. van Herk M. Different styles of image-guided radiotherapy. Semin Radiat Oncol. 2007 Oct; 17(4): 258-67. Review. 


\section{Figure}
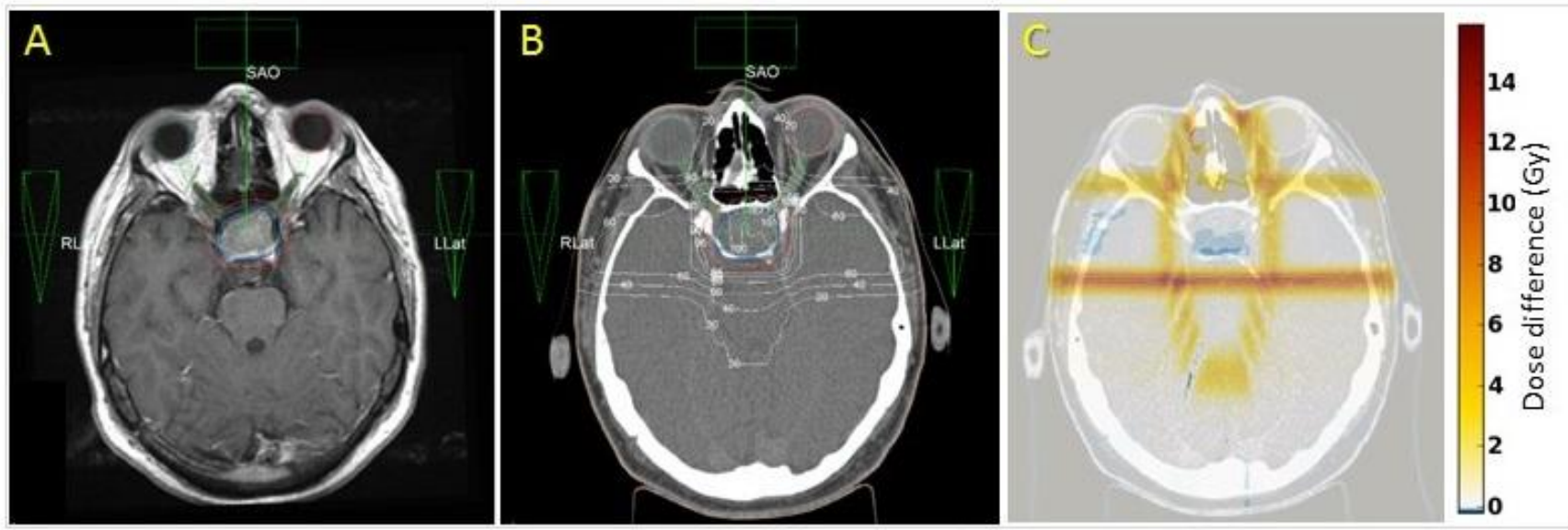

Figure. Planning MRI (A) and CT (B) scans with associated beams for a patient with recurrent pituitary adenoma. The GTV is shown in light blue, the CTV in dark blue. Two PTVs are shown: the inner (pink) has a $3 \mathrm{~mm}$ CTV to PTV margin; the outer (red) has a $5 \mathrm{~mm}$ margin. A conventional $6 \mathrm{MV}$ conformal 3 field beam arrangement has been used, with a 5 $\mathrm{mm}$ multi-leaf collimator. Isodoses for the 5mm PTV plan are shown (B). (C) shows the dose difference map, comparing the $5 \mathrm{~mm}$ PTV plan with no image guidance to a $3 \mathrm{~mm}$ PTV plan with daily image guidance, to which the daily image guidance dose has been added (i.e. Difference $=[$ Treatment dose $5 \mathrm{~mm}$ margin $]-[$ Treatment dose 3mm margin + CBCT dose $]$ ). The bands of dose difference, particularly lying posterior to the target, represent unnecessary treatment dose to which the patient would be subjected if IGRT and margin reduction are not used. 